\title{
Low-intensity pulsed ultrasound for healing supracondylar fracture
}

\author{
DOI: https://doi.org/10.5114/pq.2020.92473
}

\author{
Sara Y. Elsebahy ${ }^{1}$, Khaled A. Olama², Mohamed M. Elsayed ${ }^{3}$ \\ ${ }^{1}$ Kafrelsheikh University, Kafr El Sheikh, Egypt \\ ${ }^{2}$ Cairo University, Cairo, Egypt \\ ${ }^{3}$ Tanta University, Tanta, Egypt
}

\section{Abstract}

Introduction. Supracondylar fracture is the most common fracture in paediatrics. The present study aimed to detect the effectiveness of low-intensity pulsed ultrasound (LIPUS) on bone healing in supracondylar humeral fracture in children.

Methods. A randomized controlled study was performed. It involved 30 children suffering from supracondylar fracture, aged 5-8 years, fulfilling inclusion criteria. They were randomly allocated in 2 groups: the control group received medical treatment only and the study group underwent similar medical treatment plus LIPUS (Osteotron III ITO device), program 4, at the frequency of $3 \mathrm{MHz}$ by $160 \mathrm{~mW} / \mathrm{cm} 2$ with $20 \%$ duty cycle, at the fracture site for 20 minutes daily. Standard anteroposterior and lateral radiographs of the elbow, usually sufficient to characterize the fracture, were repeated each week until complete healing was achieved.

Results. There was a significant difference between the groups as revealed by the unpaired $t$-test. The mean value for the onset of callus was $15.86 \pm 0.74$ days in the control group and $8.4 \pm 1.35$ days in the study group, with $t=18.74$ and $p=0.0001$. The mean value of complete healing time was $34.33 \pm 1.39$ days for the control group and $21.2 \pm 3.09$ days for the study group, with $t=14.96$ and $p=0.0001$.

Conclusions. It can be concluded that LIPUS can be used effectively in promoting the healing process in patients with supracondylar fractures.

Key words: supracondylar fracture, low-intensity pulsed ultrasound, fracture healing

\section{Introduction}

Plastic deformation means that a child's bone has more elasticity than that of an adult. There are two notions that are very important: plasticity and elasticity. Bones in children are characterized by deformation characteristics before breakage so that the bone may bend but not break down [1]. The most common injury in children is supracondylar humerus fracture, responsible for approximately $1 / 3$ of fractures encountered in children and constituting the most frequent fracture involving the elbow [2]. The clinical picture of a child with supracondylar fracture involves unprompted, unceasing pain and pain worsened by palpation. There are practical restrictions in flexion and the forearm remains in semi-pronation mainly. In addition to swelling that covers the upper arm and the forearm, purplish bruising is present in the anteromedial area of the elbow [3].

There are two types of supracondylar fractures: extension and flexion type, determined by the direction of distal fragment displacement. The extension type, which accounts for approximately $97-99 \%$ of supracondylar humerus fractures, is usually caused by a falling onto an outstretched hand with the elbow in full extension position [4]. The radiographic study of the hurt limb would contain firstly an anteroposterior (AP) and then a side view of the elbow and any other places of deformity, discomfort, or tenderness [5].

Low-intensity pulsed ultrasound (LIPUS) is considered a category of ultrasound utilized for fracture treatment. It has been used to augment the healing of fracture by encouraging bone growth cells (osteogenesis) at the breakage location in order to reduce the time of improvement and accelerate the return of function [6].

LIPUS effectiveness has been observed in cases of fractures in acute stage, mal-union or fractures which delayed, stress fractures, and in children suffering from fractures that were treated by conservative methods (immobilized in a cast) or stabilized with internal or external fixation by using nails, screws, and/or plates. The impact of LIPUS as presented in the literature is intermingled and depends on the site of fracture, plan of treatment, means of measurement, and patient type [7]. Overall, LIPUS is considered the most important device, with negligible adverse events. It is labelled a gorgeous method for improving bone healing [8].

\section{Subjects and methods}

\section{Design}

The presented randomized controlled trial was conducted in an outpatient clinic of Orthopaedic Department, Faculty of Medicine, Tanta University. There were two groups of patients, randomly assigned in accordance with their referral number in the Orthopaedic Department. Patients who had an odd number were selected as the study group and those with even numbers constituted the control group. The control group received medical treatment only and the study group underwent similar medical treatment plus LIPUS (Osteotron III device), program 4, applied typically for 20 minutes per day at $3 \mathrm{MHz}, 160 \mathrm{~mW} / \mathrm{cm}^{2}, 20 \%$ duty cycle at the fracture site. Radiological assessment was performed before

Correspondence address: Sara Y. Elsebahy, Faculty of Physical Therapy, Kafrelsheikh University, Kafr El Sheikh, El-Geish street, Egypt, e-mail: drsaraelsabahy@gmail.com

Received: 14.09 .2019

Accepted: 17.11.2019

Citation: Elsebahy SY, Olama KA, Elsayed MM. Low-intensity pulsed ultrasound for healing supracondylar fracture. Physiother Quart. 2020;28(2):15-19; doi: https://doi.org/10.5114/pq.2020.92473. 
intervention and after each week until the occurrence of complete healing.

\section{Sampling}

A total of 30 children with an extension type of supracondylar fracture of humerus (Gartland type II and type III) were treated by closed manipulative reduction under general anaesthesia and percutaneous pinning. Their age ranged 5-8 years, they represented both sexes. The children took part in a randomized controlled study, and they were enrolled from the Tanta University hospitals, referred from Orthopaedic Department on the second post-operative day. The inclusion criteria were supracondylar fracture and treatment by closed reduction with pinning. Children who had pathological fractures, osteomalacia, osteoporosis, osteopenia, any musculoskeletal disorders such as mal-union, ununion, or delayed union which altered bone healing, diabetes mellitus or any vascular insufficiency disease which delayed the healing of fractures were excluded.

\section{Procedures}

Before the study, the parents of the children were explained the study purpose, details of the applied techniques, as well as the probable benefits and dangers of the study. The participants were randomly allocated into 2 groups: the control group consisted of 15 children who received medical treatment (anti-inflammatory drugs, antibiotics, and analgesia), whereas the study group consisted of 15 children who received the same medical treatment in addition to LIPUS intervention. The patients were numbered in accordance with their referral from the Orthopaedic Department; those who had an odd number were included in the study group and those with even numbers constituted the control group. All patients were subjected to the same medical treatment, and standard AP and lateral radiographs of the elbow were done before intervention and after each week until a complete union of the fracture. The lateral view should evaluate the anterior humeral line (AHL) [9], which continues the anterior cortex of the humerus. In a normal elbow, it should traverse the capitellum in its middle third (Figure 1). In a displaced fracture in extension, the AHL will pass anteriorly or may not
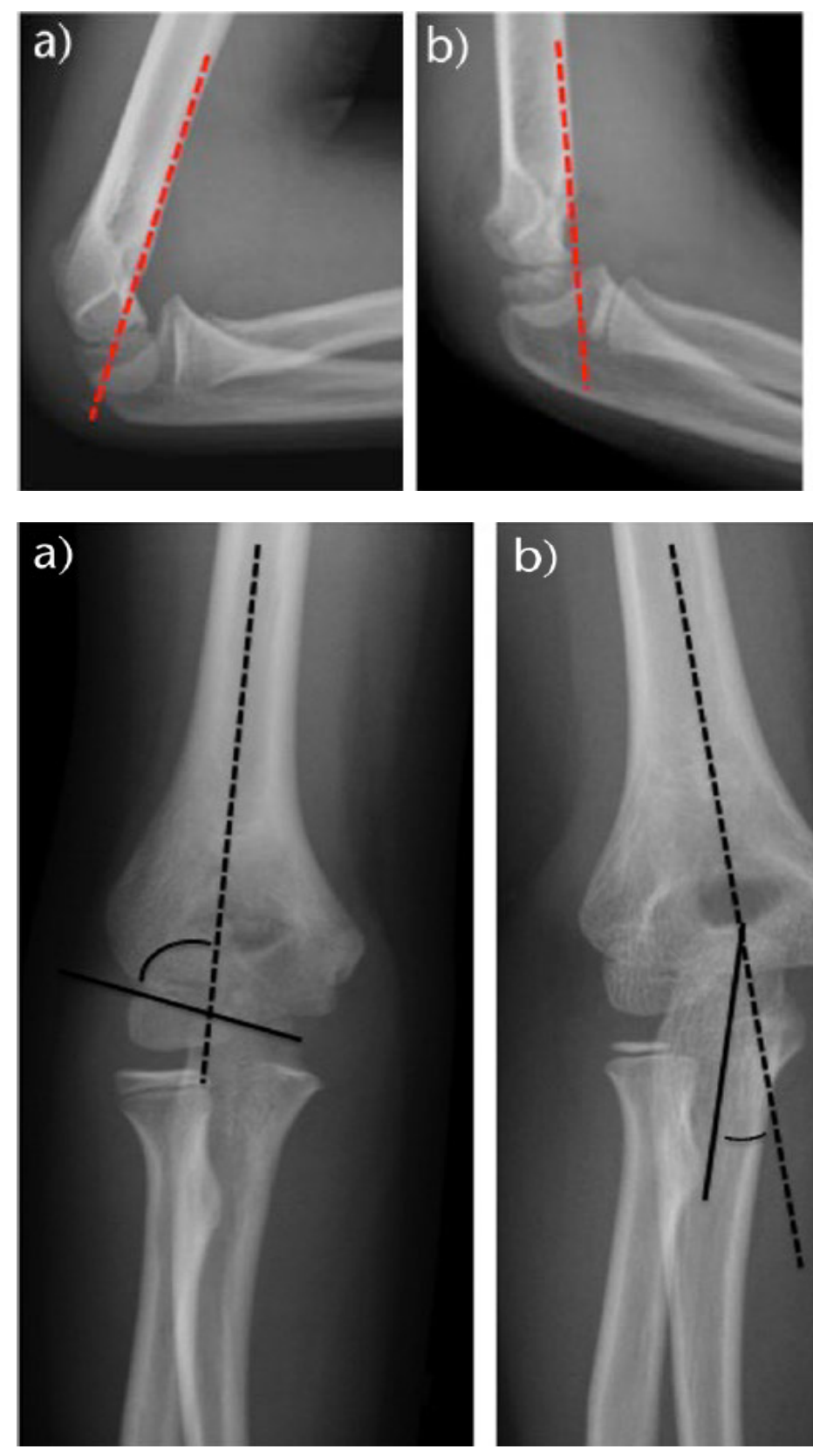

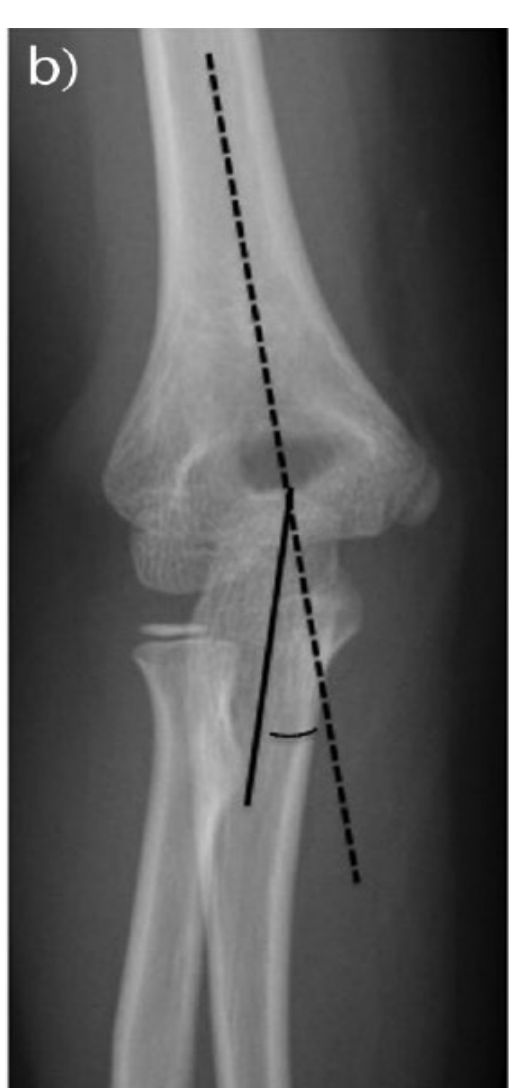

Figure 1.

(a) The anterior humeral line should traverse the middle third of the capitellum nucleus in normal anatomy and type I fractures; $(b, c)$ in extension type fractures, the line crosses anteriorly 
even cross the capitellum. In the case of a flexion type fracture, the AHL passes posteriorly to the capitellum [10]. The AP radiograph allows to evaluate the direction of displacement, the presence of varus or valgus alignment, and the extent of the fracture.

Baumann's angle is the angle formed in the AP view by the diaphyseal axis of the humerus and the physeal line of the capitellum, used to assess varus or valgus alignment of the distal humerus [11] (Figure 2).

\section{Intervention}

In the emergency department, immobilizing the elbow with a long-arm splint at $30-40^{\circ}$ of flexion is sufficient until surgery performed, in order to control pain, avoid neurovascular injuries, and minimize the risk of compartment syndrome [12]; the fracture is first reduced in the coronal plane with the elbow in extension while gentle traction is applied. When skin puckering is present because the proximal fragment is transecting the brachialis muscle [13], finally, the elbow is flexed while pushing the olecranon and maintaining the elbow in maximum flexion to stabilize the fracture until fixation with percutaneous pinning is performed [14]. It is important to note that stability is mainly provided by the pins. Fixation should be stable enough to allow immobilization of the elbow in a splint or cast in no more than about $70-80^{\circ}$ of flexion in order to avoid neurovascular complications or compartment syndrome [15]. All children received the same medical treatment (antibiotics, anti-inflammatory drugs, analgesia), but the study group started the LIPUS intervention from the $2^{\text {nd }}$ post-operative day in addition to medical treatment.

There were some general precautions that had to be observed. The child remained in a relaxed, comfortable position during the treatment. Using parts or accessories from other devices as replacements had to be avoided, and the ultrasound probe had to be handled carefully. Moreover, the following instructions were to be followed when setting up the device. The opening in cast around the fracture site, called a window, was adjusted to the LIPUS probe and made the fracture site clear. The site was cleaned carefully and gently. Gel was put on the skin of the fracture site or on sterile gauze. Then the probe was placed at the fracture site or above. LIPUS is non-invasive and can be used with pinning [16]. A portable model of ultrasound device was utilized (Osteotron III), program 4 pulsed mode, with putting gel and applying the head of the device at the fracture site directly at $3 \mathrm{MHz}$, $20 \%$ duty cycle for 20 minutes daily. The participants were instructed not to move and to keep the limb stable. After turning off the device, the area was cleaned carefully and covered. At the follow-up examination, the patients were clinically assessed for vascular status and any signs of cyanosis. X-ray imaging was performed every week to follow up the healing process. All subjects were re-evaluated after the treatment protocol; the treatment continued until complete healing occurred.

\section{Outcome measures}

These included the onset of callus formation and complete callus formation time.

\section{Power analysis}

The preliminary statistical power analysis suggested that the sample sizes of 15 participants in each group were required to achieve $80 \%$ power.

\section{Statistical analysis}

Data were first analysed by using the KolmogorovSmirnov test to recognize the normal distribution. The differences between the groups were analysed with unpaired $t$-test. The level of significance for all tests was set at 0.05 . The IBM SPSS version 21 software served to perform the statistical tests.

\section{Ethical approval}

The research related to human use has complied with all the relevant national regulations and institutional policies, has followed the tenets of the Declaration of Helsinki, and has been approved by the Ethical Committee of the Faculty of Physical Therapy, Cairo University, Egypt (No. P.T.REC/012/ 001635).

\section{Informed consent}

Informed consent has been obtained from the legal guardians of all individuals included in this study.

\section{Results}

There was a significant difference between the groups as revealed by the unpaired $t$-test. The mean value for the onset of callus was $15.86 \pm 0.74$ days in the control group and $8.4 \pm 1.35$ days in the study group $(t=18.74, p=0.0001)$. The mean value of complete healing time was $34.33 \pm 1.39$ days for the control group and $21.2 \pm 3.09$ days for the study group $(t=14.96, p=0.0001)$ (Table 1$)$.

Table 1. Mean $\pm S D, t$ and $p$ values of unpaired $t$-test in the control and study groups for onset of callus and complete healing time

\begin{tabular}{|l|c|c|c|c|}
\hline Parameter & Control group & Study group & $t$ & $p$ \\
\hline Onset of callus & $15.86 \pm 0.74$ & $8.4 \pm 1.35$ & 18.74 & $0.0001^{*}$ \\
\hline Complete callus & $34.33 \pm 1.39$ & $21.2 \pm 3.09$ & 14.96 & $0.0001^{*}$ \\
\hline
\end{tabular}

* significant values

\section{Discussion}

Harrison et al. [16] reported that there were many methods found to enhance bone healing, such as LIPUS and other non-invasive treatment options, including extracorporeal shockwave therapy, electrical and pulsed electromagnetic fields. Therefore, this study was conducted to determine the effect of using LIPUS as a non-invasive method to promote fracture healing. In 1952, Corradi and Cozzolino first stated that a continuous ultrasound wave could stimulate callus bone formation in a rabbit circular fracture model. In 1983, Duarte first applied LIPUS to stimulate bone healing in osteotomized rabbit fibulae [17]. Concurrently, Busse et al. [18] effectively used LIPUS to initiate healing of human fractures.

The purpose of the current study was to assess the improvement of healing in children with supracondylar fracture after applying LIPUS. The findings showed that LIPUS had a valuable effect on supracondylar fracture healing, which is consistent with the observations by Chaudhry et al. [19]. LIPUS enhanced bone repair processes through many mechanisms, including stimulating calcification and increasing the activity of osteoclasts with enhanced endochondral ossification. LIPUS also increased key growth factors involved in the process of angiogenesis at the fracture site, e.g. vascular endothelial growth factor A levels in osteoblasts. The produc- 
tion of fibroblast growth factor and interleukin-8 in osteoblasts was also increased. All LIPUS systems use a transducer to deliver LIPUS over the fracture site for 20 minutes [20].

The results revealed statistically significant differences between the groups with reference to the mean value of onset of callus and complete callus formation time. This remains in line with the study by Bayat et al. [21], who stated that low-level laser therapy and LIPUS treatments played a major role in the acceleration and stimulation of fracture healing and callus maturation. LIPUS is considered the most effective and these findings are very important in the evaluation of treatment with LIPUS.

These results were supported by Lee et al. [22], who revealed that there was increasing evidence that LIPUS significantly stimulated and promoted bone healing, especially in the tibia, humerus, radius, scaphoid, and other non-unions. Bayat et al. [21] performed a meta-analysis of 6 randomized controlled trials: LIPUS was found to shorten the healing time by 64 days for fractures treated conservatively as compared with the untreated control. Comparable positive effects were observed in tibial and distal radius fractures, complex tibial fractures, and scaphoid fractures [23].

Our findings also corroborated studies by Mansjur et al. [24] and Heckman et al. [25], who reported two laborious, double-blind, randomized, prospective, placebo-controlled clinical trials and assessed the rate of fracture healing. The effects of the treatment revealed that the particular ultrasound could accelerate the healing of fractures and decrease the loss of reduction during fracture healing; no serious complications were found associated with the use of ultrasound. Afterward, the effect of LIPUS on bone healing was clinically established for fresh fractures in the 1990s together with non-union cases, and LIPUS was approved by the Food and Drug Administration for the treatment of fresh fractures in 1994 and for established non-unions in 2000.

The facilitation of the healing process may be due to LIPUS promoting osteoporotic fracture healing via activating callus formation, angiogenesis and callus remodelling, involving up-regulation of Col-1, bone morphogenetic protein-2, vascular endothelial growth factor, and osteoprotegerin [26].

LIPUS waves cause nano-motion at the fracture site. The mechanical signal is converted to a biochemical signal inside the cells and is ultimately transmitted through signalling molecules to drive cyclooxygenase 2 (COX2) production in the cell. This cascade involves integrins and the formation of focal adhesions. Prostaglandin E2 production increases COX2 stimulation, which then results in the expression of osteogenic genes [27].

LIPUS has a major role in the healing of not only normal fractures but also osteoporotic fractures. This is consistent with the observations by Cheung et al. [28], who found that LIPUS showed greater results in callus width, stiffness measurement, and the response of endochondral ossification in the osteoporotic group than in the normal group in closed femoral fractures in rats.

Our study confirms the results of previous research in animal fractures [29], which proved that LIPUS accelerated bone fracture recovery and promoted the expression of osteogenic genes in rats, including bone morphogenetic protein 4, osteocalcin, and Runx2, especially at the late stage of bone repair.

Our results demonstrated acceleration and stimulation of fracture healing and callus maturation, which remains in line with the observations by Shakouri et al. [30], who found that LIPUS significantly increased callus mineral density, with an insignificant promotion in the fractured bone strength.
There were studies referring to long bones, such as femur [31], reporting that LIPUS shortened the femur fracture healing period and endochondral ossification in the process of bone healing, involving endothelial cell migration and neovascularization near fracture areas.

\section{Limitations}

The limitations of the study include the small sample size. Moreover, data difference between the sexes was not considered.

\section{Conclusions}

The findings of this study showed that LIPUS had a valuable effect on the acceleration of supracondylar fracture healing in children treated with closed and open reduction with pinning.

\section{Acknowledgments}

The authors would like to thank all participants for their contribution and support of the project.

\section{Disclosure statement}

No author has any financial interest or received any financial benefit from this research.

\section{Conflict of interest}

The authors state no conflict of interest.

\section{Significance statement}

This study introduced a favourable, non-invasive, effective method for improving healing of fractures in children that had a major role in preventing most complications that could arise from prolonged casting and immobilization, such as limitation of range of motion, stiffness, or neural complications.

\section{References}

1. Poduval M. Skeletal system anatomy in children and toddlers. 2015. Available from: http://emedicine.medscape. com/article/1899256-overview\#a.

2. Barrón-Torres EA, Sánchez-Cruz JF, Cruz-Meléndez JR. Clinical and epidemiological characteristic of humeral supracondylar fractures in pediatric patients in a Regional General Hospital [in Spanish]. Cir Cir. 2015;83(1):2934; doi: 10.1016/j.circir.2015.04.020.

3. Oetgen ME, Mirick GE, Atwater L, Lovejoy JF. Complications and predictors of need for return to the operating room in the treatment of supracondylar humerus fractures in children. Open Orthop J. 2015;9:139-142; doi: 10.2174/1874325001509010139.

4. Falciglia F, Giordano M, Aulisa AG, Di Lazzaro A, Guzzanti V. Radial neck fractures in children: results when open reduction is indicated. J Pediatr Orthop. 2014;34(8): 756-762; doi: 10.1097/BPO.0000000000000299.

5. Kumar V, Sing A. Fracture supracondylar humerus: a review. J Clin Diagn Res. 2016;10(12):RE01-RE06; doi: 10.7860/JCDR/2016/21647.8942.

6. Larking P. Low-intensity ultrasound for promoting healing of fractures. Wellington: Evidence-Based Healthcare Research Group: Accident Compensation Corporation; 2012.

7. Griffin XL, Parsons N, Costa ML, Metcalfe D. Ultrasound and shockwave therapy for acute fractures in adults. Cochrane Database Syst Rev. 2014;6:CD008579; doi: 10.1002/14651858.CD008579.pub3. 
8. Martinez de Albornoz P, Khanna A, Longo UG, Forriol F, Maffulli N. The evidence of low-intensity pulsed ultrasound for in vitro, animal and human fracture healing. Br Med Bull. 2011;100(1):39-57; doi: 10.1093/bmb/ Idr006.

9. Abzug JM, Herman MJ. Management of supracondylar humerus fractures in children: current concepts. J Am Acad Orthop Surg. 2012;20(2):69-77; doi: 10.5435/ JAAOS-20-02-069.

10. Hosseinzadeh $P$, Talwalkar VR. Compartment syndrome in children: diagnosis and management. Am J Orthop. 2016;45(1):19-22.

11. Smuin DM, Hennrikus WL. The effect of the pucker sign on outcomes of type III extension supracondylar fractures in children. J Pediatr Orthop. 2017;37(4):e229-e232; doi: 10.1097/BPO.0000000000000893.

12. Leung S, Paryavi E, Herman MJ, Sponseller PD, Abzug JM. Does the modified Gartland classification clarify decision making? J Pediatr Orthop. 2018;38(1):22-26; doi: 10.1097/BPO.0000000000000741.

13. Song MH, Kim TJ, Kang SH, Song HR. Low-intensity pulsed ultrasound enhances callus consolidation in distraction osteogenesis of the tibia by the technique of lengthening over the nail procedure. BMC Musculoskelet Disord. 2019;20(1):108; doi: 10.1186/s12891-0192490-7.

14. Carbonell R, Moraleda L, Valencia M, Diez J. Long-term functional results of pink pulseless supracondylar fractures in children when vascular injury is managed conservatively. Podium presentation, EPOSNA Annual Meeting 2017, Barcelona, Spain.

15. Prashant K, Lakhotia D, Bhattacharyya TD, Mahanta AK, Ravoof A. A comparative study of two percutaneous pinning techniques (lateral vs medial-lateral) for Gartland type III pediatric supracondylar fracture of the humerus. J Orthop Traumatol. 2016;17(3):223-229; doi: 10.1007/s10195-016-0410-2.

16. Harrison A, Lin S, Pounder N, Mikuni-Takagaki Y. Mode and mechanism of low intensity pulsed ultrasound (LIPUS) in fracture repair. Ultrasonics. 2016;70:45-52; doi: 10.1016/j.ultras.2016.03.016.

17. Leighton R, Watson JT, Giannoudis P, Papakostidis C, Harrison A, Steen RG. Healing of fracture nonunions treated with low-intensity pulsed ultrasound (LIPUS): a systematic review and meta-analysis. Injury. 2017; 48(7):1339-1347; doi: 10.1016/j.injury.2017.05.016.

18. Busse JW, Bhandari M, Einhorn TA, Schemitsch E, Heckman JD, Tornetta III P, et al. Re-evaluation of low intensity pulsed ultrasound in treatment of tibial fractures (TRUST): randomized clinical trial. BMJ. 2016;355:i5351; doi: 10.1136/bmj.i5351.

19. Chaudhry S, Rajan R, Rubin S, Papamerkouriou YM. Low intensity pulsed ultrasound (Lipus) as a non-surgical cost effective method of managing atrophic non-union. J Orthop Surg Rehabil. 2019;3(1):1-5.

20. Oei L, Rivadeneira F, Zillikens MC, Oei EHG. Diabetes, diabetic complications, and fracture risk. Curr Osteoporos Rep. 2015;13(2):106-115; doi: 10.1007/s11914015-0260-5.

21. Bayat M, Virdi A, Jalalifirouzkouhi R, Rezaei F. Comparison of effects of LLLT and LIPUS on fracture healing in animal models and patients: a systematic review. Prog Biophys Mol Biol. 2017;132:3-22; doi: 10.1016/j.pbiomolbio.2017.07.004.

22. Lee SY, Niikura T, Iwakura T, Kuroda R, Kurosaka M. Treatment of ununited femoral neck fractures in young adults using low-intensity pulsed ultrasound: report of 2 cases. Int J Surg Case Rep. 2016;21:59-62; doi: 10.1016/j.ijscr.2016.02.020.

23. Moghaddam A, Ermisch C, Schmidmaier G. Non-union current treatment concept. Shafa Ortho J. 2016;3(1): e4546; doi: 10.17795/soj-4546.

24. Mansjur KQ, Kuroda S, Izawa T, Maeda Y, Sato M, Watanabe K, et al. The effectiveness of human parathyroid hormone and low-intensity pulsed ultrasound on the fracture healing in osteoporotic bones. Ann Biomed Eng. 2016;44(8):2480-2488; doi: 10.1007/s10439-015-1533-y.

25. Heckman JD, Ryaby JP, McCabe J, Frey JJ, Kilcoyne RF. Acceleration of tibial fracture-healing by non-invasive, low-intensity pulsed ultrasound. J Bone Joint Surg Am. 1994;76(1):26-34; doi: 10.2106/00004623-19940100000004.

26. Kristiansen TK, Ryaby JP, McCabe J, Frey JJ, Roe LR. Accelerated healing of distal radial fractures with the use of specific, low-intensity ultrasound. A multicenter, prospective, randomized, double-blind, placebo-controlled study. J Bone Joint Surg Am. 1997;79(7):961-973; doi: 10.2106/00004623-199707000-00002.

27. Zura R, Mehta S, Della Rocca GJ, Jones J, Steen RG. A cohort study of 4,190 patients treated with low-intensity pulsed ultrasound (LIPUS): findings in the elderly versus all patients. BMC Musculoskelet Disord. 2015;16: 45; doi: 10.1186/s12891-015-0498-1.

28. Cheung WH, Chow SK, Sun MH, Qin L, Leung KS. Lowintensity pulsed ultrasound accelerated callus formation, angiogenesis and callus remodeling in osteoporotic fracture healing. Ultrasound Med Biol. 2011;37(2):231-238; doi: 10.1016/j.ultrasmedbio.2010.11.016.

29. Fávaro-Pípi E, Bossini $P$, de Oliveira P, Ribeiro JU, Tim C, Parizotto NA, et al. Low-intensity pulsed ultrasound produced an increase of osteogenic genes expression during the process of bone healing in rats. Ultrasound Med Biol. 2010;36(12):2057-2064; doi: 10.1016/j.ultrasmedbio. 2010.07.012.

30. Shakouri K, Eftekharsadat B, Oskuie MR, Soleimanpour J, Tarzamni MK, Salekzamani Y, et al. Effect of lowintensity pulsed ultrasound on fracture callus mineral density and flexural strength in rabbit tibial fresh fracture. J Orthop Sci. 2010;15(2):240-244; doi: 10.1007/ s00776-009-1436-6.

31. Buza JA 3rd, Einhorn T. Bone healing in 2016. Clin Cases Miner Bone Metab. 2016;13(2):101-105; doi: 10.11138/ $\mathrm{ccmbm} / 2016.13 .2 .101$. 